\title{
MS11-01 | Recalculating all X-RAy Structures in the PDB by PDB_REDO: Going
}

\section{CLOUD}

Joosten, Robbie (NKI, Amsterdam); Perrakis, Anastassis (Netherlands Cancer Institute, Amsterdam)

PDB-REDO is a refinement procedure that used REFMAC together many decision making and model building and restraints generation algorithms, to improve macromolecular structures determined by X-ray crystallography. PDBREDO is available as a web server, a software package for local installation, and - crucially - as the "back-end" for creating the PDB-REDO databank: a collection of all the PDB structures with X-ray diffraction data (well over one hundred thousand entries), re-refined and partially re-built.

To be able to offer our web services to the user community rapidly and efficiently, enable easier installation of our software, and use Grid and Cloud computing resources, PDB-REDO is available as a singularity container. This architecture has been used, among others, to re-calculate the entire PDB-REDO databank within a week in the San Diego Super-Computing center.

We will discuss the current achievement and future elans for utilising shared computing infrastructure for PDBREDO, specifically also in the context of efficient fragment-based lead discovery campaigns based on $\mathrm{X}$-ray crystallography. 